\title{
ALTERNATIVES TO THE EXISTENCE OF LARGE COOLING FLOWS
}

Wallace Tucker

Harvard-Smithsonian Center for Astrophysics, Cambridge, MA, USA

\begin{abstract}
Arguments against the existence of large scale cooling flows in clusters of galaxies are presented. The evidence for cooling flows is all circumstantial, consisting of observations of cool gas or hot gas with a radiative cooling time less than the Hubble time, or a central peak in the x-ray surface brightness profile. There is no evidence for large quantities (several tens to several hundreds of solar masses per year) of matter actually flowing anywhere. On the contrary, several lines of evidence - stellar dynamics, observations of the amount of star formation, $x$-ray surface brightness observations, theoretical calculations of the growth of thermal instabilities, the amount of cold gas - suggest that cooling flows, if they exist, must be suppressed by one to two orders of magnitude from the values implied by simple estimates based on the radiative cooling time of the $\mathrm{x}$-ray emitting gas. Two heat sources which might accomplish this - thermal conduction and relativistic particles, are considered and an alternative to the standard model for cooling flows is presented: an accretion flow with feedback wherein the accretion of gas into a massive black hole in the central galaxy generates high energy particles that heat the gas and act to limit the accretion.
\end{abstract}

“... because our descriptions of the 'real' world are metaphors based on limited abstractions from a more complex reality, it is possible to arrive at quite different, even contradictory concepts of the 'thing' which is being observed."

\section{Hanbury Brown, The Wisdom of Science}

This quotation from Hanbury Brown's wise book should be posted in every scientist's office as a constant reminder that we are dealing with metaphors based on limited abstractions from limited data on a very complex reality. Cooling flows are a case in point. From the same data it is possible, by changing what is assumed about the unknown, to arrive at almost contradictory pictures of what is being observed. Hopefully in the not too distance future high resolution x-ray spectroscopy 
will identify the set of unknowns so that the seemingly contradictory pictures or metaphors can be revised and reconciled into one that more accurately reflects the richness and complexity of the underlying reality.

\section{COOLING FLOWS - THE STANDARD METAPHOR}

The standard cooling flow metaphor has been well reviewed by Andy Fabian (1988). The key observations are high resolution x-ray surface brightness profiles and spectral measurements which demonstrate conclusively that the radiative cooling time inside a certain critical radius, called the cooling radius, in the central regions of clusters of galaxies is shorter that the assumed cluster lifetime of 10 to 20 billion years. Under the assumption that no significant heat sources are present, the observations can be taken to mean that radiative cooling is driving mass accretion onto a stationary supergiant galaxy at a rate given by

$$
\dot{M}_{o}=\frac{4 \pi r^{2} n^{2} \wedge(t)}{\frac{d}{d r}\left(\frac{5}{2} \frac{k T}{\mu m_{p}}+\phi\right)} \approx \frac{2 \mu L_{x}}{5\left(k / m_{p}\right) T} \mathrm{gm} / \mathrm{sec}
$$

where $r$ is the radius, $n$ the electron density, $\wedge(T)$ is the plasma emissivity at temperature $T, k$ is Boltzmann's constant, $\mu$ is the mean molecular weight, $m_{p}$ is the proton mass, $\phi(r)$ is the gravitational potential and $L_{x}$ is the x-ray luminosity. From this equation, values of the accretion rate ranging from several to several hundred solar masses per year have been derived for a wide variety of cluster types (Sarazin 1986, Mushotzky and Szymkowiak 1988, Arnaud and Fabian 1988).

\section{PROBLEMS WITH THE STANDARD METAPHOR}

These large accretion rates pose a vexing problem: what has become of the tens of billions to trillions of solar masses of accreted gas implied by these estimates? It has been argued that the optical observation of $\mathrm{H}$-alpha filamentary structures is evidence in favor of these large cooling rates. However, neither the mass in these filaments, nor the $\mathrm{H}$-alpha emissivity is consistent with the predictions of the standard cooling flow model. The mass is rather small, on the order of a million solar masses and the filaments require an additional heat source to explain their emissivity. The connection of the optical filaments to the cooling $\mathrm{x}$-ray gas is indirect at best. Their existence certainly cannot be taken as evidence that massive cooling flows are occurring.

The cooling gas does not show up in radio observations of $\mathrm{HI}$ at $21 \mathrm{~cm}$, so it cannot be hidden in cold gas either. Nor can it be in black holes in the nuclei of the central galaxies, for it would be detectable both through its effects on the stellar dynamics of the galaxy (Mathews 1988) and through the energy released by accretion of several solar masses of gas per year into the black hole:

$$
L_{a c c} \approx 6 \times 10^{45} \dot{m}_{0} \mathrm{erg} / \mathrm{s}
$$


where $\dot{m}_{0}$ is the accretion rate in solar masses per year.

Finally, the accreting mass cannot have gone into the formation of a normal stellar population. Otherwise, the rate of star formation would be inconsistent with the observed colors of the central galaxies. O'Connell and McNamara (1988) have shown that evidence for star formation does exist in about $50 \%$ of the central galaxies in cooling flow clusters, but a rate that is less than $10 \%$ and more often less than $1 \%$ of the level implied by the accretion rates derived in the standard model. The implication of their work is that, if the accreted mass goes into the formation of stars, it must be an invisible population with an average mass $<<0.7$ solar masses.

Several authors have suggested that the accreted mass is going into just such a population (see, e.g., Sarazin and O'Connell 1983, Fabian et al. 1982). They argue that the formation of loss mass stars is expected in cooling flows because the high pressures imply small values of the Jeans mass (proportional to $T^{2} p^{-1 / 2}$ for a protostellar cloud of temperature $T$ and pressure $p$ ). The observation that $\dot{m}_{0}$ is not constant, but increases with radius roughly as $\dot{m}_{0} \propto r$, has been taken as evidence that matter is dropping out of the flow (Fabian 1988a,b), presumably to form a population of very low mass stars.

However, several theoretical treatments of the problem of matter condensing out of the flow indicate that it cannot occur in such a way as to explain the observed $\dot{m}_{0}$ versus $r$ dependence unless ad hoc assumptions are made concerning the existence and distribution of large, pre-existing clumps of matter in the cooling flow (Balbus and Soker 1988, Malagoli, Rosner, and Bodo 1987, Nulsen 1988). Further, it has been shown the pressures in accretion flows are comparable to those in dense molecular clouds, which are thought to be some of the most prolific sites of star formation in our galaxy, and stars of a solar mass or higher have no difficulty forming there (Scalo and Miller 1979). The possibility of hiding large amounts of cooling matter from accretion flows in low mass stars remains speculative, with no empirical basis.

\section{AN ALTERNATE METAPHOR - AN ACCRETION FLOW WITH A HEAT- ING FEEDBACK MECHANISM}

In view of the difficulties encountered with the standard model, it seems worthwhile to consider an alternate model in which we drop the assumption that no heat sources exist (Tucker and Rosner 1983, Rosner and Tucker 1989). In the presence of a heat source $q_{H}$, the accretion rate is

$$
\dot{m}_{a}=\dot{m}_{0}\left\{1-\left[q_{H} / n^{2} \wedge(T)\right]\right\}
$$

Where $\dot{m}_{a}$ is the actual accretion rate, as contrasted with $\dot{m}_{0}$, calculated from equation (1), which is the apparent heating rate, derived on the assumption that no heat sources are present. This equation shows that the accretion rate can be arbitrarily small for an arbitrarily fine balance between heating and radiative losses. This 
provides a simple solution to the problem of hiding excessive amounts of accreted mass: very little mass is actually accreted, so there is little or no mass to hide.

The existence of a heat source can also resolve the paradox posed by the observation that $\dot{m}_{0}$ varies with radius, whereas the theoretical calculations indicate that it should show little or no variation. If equation (3) is re-written in the form

$$
\dot{m}_{0}=\dot{m}_{a} /\left[1-\left(q_{H} / n^{2} \wedge(T)\right)\right]
$$

it is apparent that, if $q_{H} / n^{2} \wedge(T)$ is a function of radius, then the apparent accretion rate $\dot{m}_{0}$ will be a function of radius, even though the actual accretion rate $\dot{m}_{a}$ is constant.

Of course, this resolution of the difficulties of the standard model is academic if no acceptable heat sources exist. On the one hand, Fabian (1988b) has maintained at this symposium that this is the case. On the other hand, I contend that sufficiently strong heat sources do exist in many if not all of the cooling flow clusters and present evidence below to support this contention.

\section{ESTIMATES OF THE IMPORTANCE OF HEATING BY THERMAL CON- DUCTION}

The importance of thermal conduction as a heat source in clusters of galaxies has been recognized by a number of authors (Rephaeli 1977, Tucker and Rosner 1983, Bertschinger and Meiksen 1986, Friaca 1986, Volkov 1985). We can estimate the strength of thermal conduction relative to radiative losses by taking the ratio of the divergence of the conductive heat flux with the radiative energy losses at the cooling radius, that is, the radius at which the radiative cooling time $=10^{10}$ years. This ratio, which $\mathrm{I}$ call $\mathrm{C}$, is given by

$$
C=q_{\text {cond }} / n^{2} \wedge(T)=0.5 T_{7}^{2} b / r_{100}^{z}
$$

where $r_{100}$ is the cooling radius in units of $100 \mathrm{kpc}, T_{7}$ is the temperature in units of $10^{7}$ degrees, and $b$ represents the factor by which thermal conduction is reduced by magnetic field effects. Rosner and Tucker have given arguments that this factor should not be smaller than about 0.2 , so I take $b=0.2$.

The accompanying table shows the computed values for $\mathrm{C}$ for the cooling flow clusters listed in the catalog of Arnaud and Fabian (1989). The values of the temperature and cooling radius derived by Arnaud and Fabian from the observations are used in the computation, so $\mathrm{C}$ is an empirical, or at least, semi-empirical estimate of the importance of thermal conduction. Of the 45 clusters in the table, 32 have $C>1$, indicating that thermal conduction should be considered as a major heat source for the majority of cooling flow clusters. The effect of $C>1$ is to shift the cooling radius inward from the value predicted by the standard model, to change the temperature profile, making it flatter at smaller radii, and to reduce 
the accretion rate. The temperature profiles are not sufficiently well known to determine observationally just how important thermal conduction is. This is an area where high-resolution $\mathrm{x}$-ray spectroscopy can make a crucial contribution.

One interesting suggestion, which literally turns the standard model insideout, is that in Centaurus and possibly other clusters, we observing, not a cooling inflow, but an evaporative outflow of gas (de Jong et al. 1988). If this were the case, then the gas supply would have to be replenished at an average rate of several solar masses per year or more. de Jong et al. suggest that this could be accomplished by collisions and stripping of galactic halos in the central regions of rich clusters.

\section{ESTIMATES OF THE IMPORTANCE OF HEATING BY RELATIVISTIC PARTICLES}

Another potentially important heat source in cooling flow clusters is relativistic particles (Sofia 1973, Lea and Holman 1978, Rephaeli 1979, Scott et al 1980, Tucker and Rosner 1983, Pedlar et al. 1988, Miller 1988). Using the expression derived by Scott et al. for heating by relativistic plasma processes, together with the estimates of relativistic electron density given by Odea and Baum (1986) and the data on the hot gas from Arnaud and Fabian (1989), the ratio $\mathrm{H}$ of heating by relativistic electrons to radiative cooling of the hot gas has been estimated for 21 sources studied by Odea and Baum (1986). The results, shown in Table 1, indicate that relativistic electron heating is important in 12 of these sources, is probably not important in 5 sources, and the data is inconclusive in 4 sources.

In only one source is the sum $C+H<1$ : MKW 4, a poor cluster with a central dominant galaxy. MKW 4 is the best candidate to be standard cooling flows in which no heating is occurring. Detailed $x$-ray spectroscopic observations of this source would be especially interesting. In particular it would be interesting to know if the accretion rate is constant with radius, as the standard model in its simplest form predicts.

\section{COOLING FLOWS WITH FEEDBACK}

The data in Table 1 indicate that we must take seriously the possibility that heating by conduction and relativistic particles modifies the standard cooling flow model. If thermal conduction is sufficiently strong, it will make the cluster gas virtually isothermal or it may even drive an evaporative wind. In many cases, conduction will not shut off the accretion rate, completely, but will move the cooling radius inward and reduce the accretion rate by a factor of two or three. In these cases, a feedback mechanism involving accretion and heating by relativistic particle may come into play, reducing by accretion rate by a large factor.

An alternative scenario for cooling flows might go as follows: radiative cooling in the central regions lead to pressure gradients and an accretion flow. A portion 
TABLE 1

Estimates of the Importance of Heating by Thermal Conduction and Relativistic Particles

\begin{tabular}{|c|c|c|}
\hline Source & $\begin{array}{c}\mathrm{C} \\
\left(=q_{\text {cond }} / n^{2} \wedge(T)\right)\end{array}$ & $\begin{array}{c}\mathrm{H} \\
\left(=q^{\text {rel }} / n^{2} \wedge(T)\right)\end{array}$ \\
\hline SC0004 & 3.0 & $?$ \\
\hline A85 & 4.8 & 7.2 \\
\hline A133 & 1.1 & $?$ \\
\hline A262 & 0.2 & 1.2 \\
\hline A278 & 4.3 & $?$ \\
\hline A376 & 1.1 & $?$ \\
\hline AWM 7 & 1.4 & $<0.3$ \\
\hline A401 & 18.0 & $?$ \\
\hline A426 Perseus & 3.3 & 8.5 \\
\hline A 478 & 0.8 & $?$ \\
\hline $\mathrm{A} 496$ & 1. & $<0.6$ \\
\hline A539 & 2.2 & $?$ \\
\hline A576 & 5.0 & $<6.7$ \\
\hline A592 & 3.0 & $?$ \\
\hline PKS0745 & 2.5 & 1123 \\
\hline A644 & 0.5 & $?$ \\
\hline A665 & 1.4 & $?$ \\
\hline A 1060 & 0.5 & $<0.9$ \\
\hline MKW 4 & 0.2 & $<0.02$ \\
\hline Centaurus & 0.4 & $?$ \\
\hline A1644 & 2.5 & $?$ \\
\hline A1689 & 2.5 & $?$ \\
\hline A1767 & 1.4 & $?$ \\
\hline A 1795 & 1.1 & 113 \\
\hline A 1877 & 1.9 & $?$ \\
\hline $3 \mathrm{C} 295$ & 1.8 & $<25$ \\
\hline A1983 & 9.8 & $?$ \\
\hline A1991 & 1.2 & 14 \\
\hline A2029 & 1.5 & 6 \\
\hline A2052 & 0.3 & $?$ \\
\hline A2063 & 1.0 & $?$ \\
\hline MKW 3s & 0.2 & $?$ \\
\hline A2142 & 3.3 & $<0$ \\
\hline A2151 & 1.3 & $?$ \\
\hline AWM 4 & 0.6 & 137 \\
\hline A2199 & 0.5 & 208 \\
\hline Her A & 0.1 & $?$ \\
\hline A2244 & 1.2 & $?$ \\
\hline $\mathrm{SC} 184$ & 27.8 & $?$ \\
\hline Cyg A & 1.5 & $?$ \\
\hline A2415 & 2.2 & $<615$ \\
\hline
\end{tabular}


of the accreted matter falls into a black hole in the nucleus of the central galaxy, thereby generating nuclear activity, some of which appears in the form of relativistic particles. These relativistic particles stream out into the gaseous halo, heating it and reducing the accretion rate, which after a certain time delay $\tau$ reduces the relativistic particle production.

The equation for the accretion rate then becomes

$$
\dot{m}_{a}(t)=\dot{m}_{0}-\beta \dot{m}(t-\tau)
$$

where $m_{0}$ is given by equation (1),

$$
\beta=\alpha m_{p} c^{2} / k T \approx 10^{6} \alpha / T_{7}
$$

and $\alpha$ is a factor that takes into account the efficiency of the accretion process in producing relativistic particles. The steady state solution to equation (5) is

$$
\dot{m}_{s s}=\dot{m}_{0} /(1+\beta)
$$

It may be that the system does not reach a steady state, but exhibits limit cycle behavior, oscillating between relatively long-lived states of no accretion and short-lived states of accretion rates near $m_{0}$, so that the time-averaged accretion rate is close to the value given by equation (7).

Finally, another byproduct of the model is that it may be ale to explain the optical filaments. As discussed by Bohringer and Morfill (1988), a large relativistic electron population can lead to a large non-thermal pressure, which can in turn lead to Rayleigh-Taylor instabilities. These instabilities could be the generators of the optical filaments, and the relativistic particles could supply the missing heat source.

\section{ACKNOWLEDGEMENTS}

I thank K. Arnaud and A. Fabian for making the data in their cooling flow catalog available prior to publication. I also thank Carolyn Stern for her computational assistance and W. Forman, C. Jones and R. Rosner for helpful discussions. This work was supported by NAS8-3075.

\section{REFERENCES}

Arnaud, K. and Fabian, A. 1988, in preparation.

Balbus, S. and Soker, N. 1988, this symposium.

Bertschinger, E. and Meiksen, A. 1986, Ap.J., 306, L1.

Bohringer, H. and Morfill, G. 1988, Ap.J., 330, 609.

de Jong, T., Norgaard-Nielsen, H., Jorgensen, H., and Hansen, L. 1988, preprint.

Fabian, A. 1988a, in Cooling Flows in Clusters and Galaxies, ed. A. Fabian (Kluwer Academic Publishers, Dordrecht). 
Fabian, A. 1988b, this symposium.

Fabian, A., Nulsen, P. and Canizares, C. 1982, MNRAS, $201,933$.

Friaca, A. 1986, Astron.Ap., 164, 6.

Lea, S. and Holman, G. 1978, Ap.J., 222, 29.

Malogoli, A., Rosner, R. and Bodo, G. 1987, Ap.J., 319, 622.

Mathews, W. 1988, in Cooling Flows in Clusters and Galaxies, ed. A. Fabian (Kluwer Academic Publishers, Dordrecht).

Miller, L. 1988, in Cooling Flows in Clusters and Galaxies, ed. A. Fabian (Kluwer Academic Publishers, Dordrecht).

Mushotzky, R. and Szymkowiak, A. 1988, in Cooling Flows in Clusters and Galaxies, ed. A. Fabian (Kluwer Academic Publishers, Dordrecht).

Nelsen P. 1988, in Cooling Flows in Clusters and Galaxies, ed. A. Fabian (Kluwer Academic Publishers, Dordrecht).

O'Connell, R. and McNamara B. 1988, in Cooling Flows in Clusters and Galaxies, ed. A. Fabian (Kluwer Academic Publishers, Dordrecht).

DISCUSSION-W. Tucker

C. CANIZARES: Andy Fabian has already noted that the SSS and FPCS have detected $x$-ray emission lines from approximately 10 clusters. The values for the accretion rate deduced from these lines agree with those derived from the $\mathrm{x}$-ray images, but leaving that aside, the lines indicate the presence of large quantities of relatively cool gas. These are crucial observations that must be explained by any model. The thermal conduction models fail to do this (at least those in the literature). How do the heating models do?

W. TUCKER: In the relativistic particles heating model described here, the balance between heating and cooling requires, roughly, that the temperature $T \propto 1 / p^{\text {rel }}$, where $p^{\text {rel }}$ is the relativistic particle pressure. So, for example, if $p^{r e l} \propto 1 / r$, then $T \propto r$, inside the cooling radius, in rough agreement with the observations.

J. CULHANE: Can you estimate a characteristic time for the cycle of cooling flow followed by a heat input due to particles? Presumably there will be radio emission while the particle source is on, so this might have observational consequences.

W. TUCKER: The cycle will be composed of at least three parts:

(1) cooling and accretion on a time scale of $10^{9}-10^{10}$ years;

(2) production and diffusion of electrons throughout the source on a time scale of about $10^{8}$ years;

(3) decay of the electron population on a time scale of $10^{8}-10^{9}$ years. 